\title{
Methylthioadenosine phosphorylase deficiency in Japanese osteosarcoma patients
}

\author{
SHINICHI MIYAZAKI ${ }^{1}$, JUNJI NISHIOKA ${ }^{2}$, TAIZOU SHIRAISHI $^{3}$, \\ AKIHIKO MATSUMINE $^{1}$, ATSUMASA UCHIDA ${ }^{1}$ and TSUTOMU NOBORI ${ }^{2}$ \\ Departments of ${ }^{1}$ Orthopaedic Surgery, and ${ }^{2}$ Molecular and Laboratory Medicine, \\ ${ }^{3}$ Second Department of Pathology, Mie University School of Medicine, Mie, Japan
}

Received May 9, 2007; Accepted July 12, 2007

\begin{abstract}
Methylthioadenosine phosphorylase (MTAP) is an important enzyme in the salvage pathway of adenosine and methionine synthesis. MTAP is ubiquitously present in all normal cells and tissues, but deficient in a variety of malignant tumors. The enzyme deficiency is caused by either MTAP gene deletion or promoter hypermethylation. We investigated MTAP expression, MTAP gene deletion and promoter abnormality in 40 primary tumor samples from Japanese osteosarcoma patients and determined the frequency of the enzyme deficiency. We also tested whether or not the enzyme deficiency can be exploited for tumor-specific chemotherapy using osteosarcoma cell lines. For MTAP expression, immunohistochemistry (IHC) and Western blotting were used. Realtime quantitative PCR assay was used for the analysis of MTAP gene deletion in fifteen osteosarcoma samples. MTAP promoter abnormality was analyzed by methylation-specific PCR. Then, the relationship between MTAP expression and sensitivity to the inhibitors of de novo AMP synthesis was confirmed in an MTAP-negative and -positive osteosarcoma cell line. The MTAP protein was negative in 11 of 40 samples (27.5\%) by IHC and in 4 of 6 osteosarcoma cell lines (66.7\%) by Western blot analysis. Among 40 samples, 15 were subjected to quantitative real-time PCR and promoter methylation analysis. Of 6 samples that were negative by IHC, the MTAP gene was deleted in 3 and the MTAP promoter was methylated in 2. These results indicated that MTAP deficiency was caused by MTAP gene deletion or promoter methylation in all MTAPnegative samples except one that was negative with IHC although no deletion or promoter methylation was detected. In in vitro experiments using transfectoma along with the MTAP-negative parental cell line, the MTAP-negative parental cell line was more chemosensitive to the inhibitors of de novo
\end{abstract}

Correspondence to: Dr Tsutomu Nobori, Department of Molecular and Laboratory Medicine, Mie University School of Medicine, 2-174 Edobashi, Tsu-city, Mie 514-8507, Japan

E-mail: nobori@clin.medic.mie-u.ac.jp

Key words: methylthioadenosine phosphorylase, osteosarcoma, immunohistochemistry, chemosensitivity
AMP synthesis than MTAP-positive transfectoma. MTAP deficiency frequently found in osteosarcoma can be exploited for selective chemotherapy in MTAP-negative osteosarcoma patients with the inhibitors of de novo purine synthesis.

\section{Introduction}

Osteosarcoma is the most common bone tumor in children and accounts for $50 \%$ of all primary bone tumors. Over 100 patients are newly diagnosed with the disease annually in Japan. The prognosis of osteosarcoma was poor until adjuvant and neoadjuvant chemotherapy was commonly used. Although the 10-year disease-free survival is approximately $60 \%$ in patients with localized disease, it is still low in patients with metastatic disease at diagnosis (1).

Methylthioadenosine phosphorylase (MTAP) is ubiquitously expressed in all normal tissues (2). MTAP catalyzes the phosphorolysis of the nucleoside MTA to adenine and methylthioribose-1-phosphate (MTR-1-P), which are salvaged to AMP and methionine, respectively (3). The MTAP gene consists of 8 exons (4) and is on chromosome 9 p21 in close proximity to the tumor suppressor genes $\mathrm{p} 16^{\mathrm{INK} 4 \mathrm{a}}$ and $\mathrm{p} 15^{\mathrm{INK} 4 \mathrm{~b}}(5,6)$. MTAP has been reported to be frequently deficient in a variety of malignant tumors including leukemia, glioma, non-small cell lung cancer, bladder cancer, malignant melanoma, pancreatic cancer, adult T-cell leukemia (ATL) and osteosarcoma (7-17). MTAP deficiency was mainly caused by MTAP gene deletion (4). In recent reports, MTAP deficiency was caused by gene silencing through an epigenetic mechanism including promoter hypermethylation in the cultured cell line as well as primary tumors $(18,19)$.

Since the salvage of adenine and methionine from MTA is blocked in malignant cells lacking the MTAP enzyme, AMP synthesis is completely dependent on the de novo pathway. Accordingly, the MTAP-negative cells are sensitive to the inhibitors of AMP synthesis, and MTAP deficiency can be exploited to selectively treat MTAP-negative tumors $(9,20-23)$.

Although several diagnostic methods have been used to detect MTAP gene deletion in malignant tumors, the precise diagnosis of MTAP gene deletion has been hampered by the presence of contaminating normal cells $(8,24)$. We developed the real-time polymerase chain reaction (RT-PCR) assay 
Table I. Clinical characteristics of 40 patients with osteosarcoma.

\begin{tabular}{lc}
\hline Characteristics & Number of patients $(\%)$ \\
\hline Age & \\
6-79 years (median:17.5) & 40 \\
Gender & $21(52.5)$ \\
Male & $19(47.5)$ \\
Female & \\
Location & $22(55.0)$ \\
Femur & $7(17.5)$ \\
Tibia & $5(12.5)$ \\
Humerus & $2(5.0)$ \\
Fibula & $4(10.0)$ \\
Other & \\
Histology & $22(55.0)$ \\
Osteoblastic & $10(25.0)$ \\
Chondroblastic & $5(12.5)$ \\
Fibroblastic & $3(7.5)$ \\
Telangiectatic & \\
Metastasis & $6(15.0)$ \\
Present & $34(85.0)$ \\
Absent & \\
\hline
\end{tabular}

using TaqMan chemistry (Perkin Elmer-Applied Biosystems ABI Prism 7700 sequence detection system) (25).

In previous reports, the MTAP-negative malignant cell lines were shown to be more chemosensitive to the inhibitors of de novo AMP synthesis than MTAP-positive cell lines $(9,15,22,26)$. We transformed the MTAP-negative osteosarcoma cell lines to express MTAP and assessed the effects of the MTAP expression on chemosensitivity in vitro.

The aim of the present study was to analyze the MTAP expression in osteosarcoma patients and cell lines and examine the relationship between MTAP expression and chemosensitivity for the inhibitors of AMP synthesis.

\section{Materials and methods}

Patient samples. Forty osteosarcoma samples were obtained from consenting patients who underwent surgery at the Department of Orthopaedic Surgery, Mie University Hospital from 1989 to 2003 (Table I). All samples were paraffinembedded following decalcification.

Cell lines. Six human osteosarcoma cell lines were used: U2OS, HOS, KHOS, G292 and MG63 from the American Type Culture Collection (ATCC), and SaOS-2 from Cell Resource Center for Biomedical Research Institute of Development, Aging and Cancer, Tohoku University. These cell lines were grown in Dulbecco's modified Eagle's medium (Gibco-BRL Laboratory, Grand Island, NY, USA) containing 10\% fetal bovine serum at $37^{\circ} \mathrm{C}$ in humidified $5 \% \mathrm{CO}_{2}$ and $95 \%$ air.
MTAP expression vector. To construct the MTAP expression vector, the human MTAP cDNA was cut out with EcoRI and XhoI enzyme digestion, and ligated into the pcDNA3.1/Zeo(+) expression vector (Invitrogen, Carlsbad, CA, USA). The resultant plasmid DNA was extracted with the HiSpeed ${ }^{\text {TM }}$ Plasmid Maxi kit according to the manufacturer's protocol (Qiagen, Gmb., Hilden, Germany).

Transfection with MTAP expression vector. The MTAPnegative osteosarcoma cell lines (HOS, KHOS, G292 and MG63) were transfected with Lipofectamine 2000 (Invitrogen) in growth medium according to the supplier's instructions. After transfection with MTAP expression vector, the cells were diluted at 1:10 and were selected in DMEM containing Zeocin (Invitrogen) at $400 \mu \mathrm{g} / \mathrm{ml}$. Following incubation for 2 weeks in the presence of Zeocin, the grown cells were collected and subjected to cloning by the limiting dilution method.

Chemotherapeutic agents. MTX was kindly supplied by Weyth-Lederle, Japan. L-alanosine was kindly supplied by Dr. Lorenzo Leoni (Salmedix, Inc., San Diego, CA, USA).

The monoclonal antibody against human MTAP. The antihuman MTAP monoclonal antibody was produced by the procedure described by Galfre and Milstein (27). Briefly, the splenic cells obtained from BALB/c mice injected with recombinant MTAP protein were used to produce hybridomas, which were screened for the antibody production by ELISA with recombinant MTAP protein as an antigen. Positive hybridomas were cloned by the limiting dilution method. The antibody-producing clones were propagated as ascitic fluid in mice by the procedure of Harlow and Lane (28). The isotyping of monoclonal antibody was performed with a mouse monoclonal isotyping kit (mouse immunoglobulin isotyping CBA; BD PharMingen, San Diego, CA, USA) according to the manufacturer's instructions. Ascitic fluid was concentrated by ammonium sulfate precipitation, and the immunogloblin $G$ (IgG) fraction was purified by using protein A column (27).

Immunohistochemistry. Immunohistochemistry (IHC) was achieved with the Ventana EX system using a DAB universal kit (Ventana Medical Systems, Inc., Tucson, AZ, USA). Paraffin-embedded osteosarcoma tissue was cut into 4- $\mu$ m thick sections, placed on silanized slides (Dako-Japan, Kyoto, Japan) and immunohistochemically stained by using the streptavidinbiotinylated complex method. The sections were deparaffinized in xylene and rehydated in ethanol. Antigen retrieval was performed with $4.0 \mathrm{mM}$ citric acid buffer, using a Panasonic microwave at the high setting 3 times for $25 \mathrm{sec}$. After cooling for $30 \mathrm{~min}$, the sections were placed in a VENTANA autostainer. The mouse monoclonal anti-MTAP antibody generated in our laboratory as described above was diluted at 1:100 in antibody diluent buffer (Dako-Japan). Plates were reacted with the secondary antibody (anti-mouse IgG antibody), followed by coloration with 3-3' diaminobenzidine tetrahydrochloride (DAB). Hematoxylin was used for the final nuclear counterstaining. Results were evaluated based on the cytoplasmic staining density in each cell and the immunostained area of the magnified field. When the immunostaining 
density was comparable to that of normal alveolar cells used as a control and the immunostained area exceeded $25 \%$ of the magnified field, the specimen was regarded as positive. When the immunostained area was $<25 \%$, the specimen was regarded as negative. All stained slides were microscopically observed with x400 magnification and examined independently by two pathologists.

Western blot analysis. Osteosarcoma cell lines were cultured to sub-confluence in 100-mm dishes containing growth medium. After washing with phosphate-buffered saline (PBS), cells were collected using a cell scraper and lysed in RIPA buffer (10 mM Tris-HCl, pH 7.4, 1\% NP-40, 0.1\% sodium deoxycholate, $0.1 \%$ sodium dodecyl sulfate, $0.15 \mathrm{M} \mathrm{NaCl}$, $1 \mathrm{mM}$ EDTA, $10 \mu \mathrm{g} / \mathrm{ml}$ aprotinin).The protein concentration was measured using the BCA protein assay reagent kit (Pierce, Rockford, IL, USA). The $25 \mu \mathrm{l}$ aliquots $(20 \mu \mathrm{g})$ of the extracted samples were boiled following the addition of $50 \mu \mathrm{l}$ of $6 \mathrm{X}$ sample buffer (1X buffer: $50 \mathrm{mM}$ Tris-HCl, $\mathrm{pH} 6.8,10 \%$ glycerol, $2 \%$ sodium dodecylsulfate, $100 \mathrm{mM}$ dithiothreitol, $0.1 \%$ bromophenol blue) and were resolved by electrophoresis on $10 \%$ polyacrylamide gels containing $0.1 \%$ SDS. Gels were soaked for $30 \mathrm{~min}$ in transfer buffer (running buffer containing $15 \%$ methanol) and then blotted onto polyvinylidene difluoride (PVDF) membranes (Millipore, Bedford, MA, USA) using a Bio-Rad transfer apparatus. The membrane was washed in Tris buffered saline containing $0.1 \%$ Tween-20 (TBS-T) and incubated with the mouse monoclonal anti-MTAP antibody (1:250) overnight at $4^{\circ} \mathrm{C}$. The membrane was washed three times with TBS-T, and MTAP was visualized using a goat anti-mouse antibody labeled with peroxidase (1:5000) and NBIC/NST substrate (Kirkegaard \& Perry Laboratory, Gaithersburg, MD, USA).

Analysis of MTAP gene deletion. MTAP gene deletion was analyzed by quantitative RT-PCR as previously described (25). Genomic DNA was extracted from six osteosarcoma cell lines and primary tumor specimens stored in DF medium (Nissui Phamaceutical, Tokyo, Japan) at $-80^{\circ} \mathrm{C}$ using Sepa Gene (Sankou Junyaku, Tokyo, Japan). Of 40 osteosarcoma patients, tumor specimens sufficient for DNA extraction were available from 15. A PCR assay was performed with 6 cell lines and 15 tumor samples.

Methylation-specific PCR. Methylation-specific PCR analysis was performed when an immunoreactive MTAP protein was not observed with IHC although the MTAP gene was intact. Genomic DNA was treated with sodium bisulfite as described (29). Briefly, DNA ( $2 \mu \mathrm{g}$ ) was denatured by sodium hydroxide at a final concentration of $0.2 \mathrm{M}$ for $10 \mathrm{~min}$ at $37^{\circ} \mathrm{C}$. The denatured DNA was added with $30 \mu 1$ of $10 \mathrm{mM}$ hydroquinone (Sigma, St. Louis, MO, USA) and $520 \mu 1$ of $3.6 \mathrm{M}$ sodium bisulfite (Sigma) at pH 5.0. Samples were incubated overnight at $55^{\circ} \mathrm{C}$. Modified DNA was purified using the Wizard DNA Clean-up system (Promega, Madison, WI, USA), and the chemical reaction was terminated by adding sodium hydroxide at a final concentration of $0.3 \mathrm{M}$, followed by incubation for $5 \mathrm{~min}$ at room temperature. After ethanol precipitation, DNA was resuspended in $25 \mu 1$ of distilled water and stored at $-20^{\circ} \mathrm{C}$ until use.
Table II. Correlation between MTAP expression and clinical characteristics in osteosarcoma.

\begin{tabular}{lcc}
\hline Characteristics & \multicolumn{2}{c}{ MTAP $^{\mathrm{a}}$} \\
\cline { 2 - 3 } & Positive & Negative \\
\hline Age (years) & 17 & 8 \\
$<20$ & 12 & 3 \\
$\geq 20$ & & \\
Gender & 18 & 3 \\
Male & 11 & 8 \\
Female & & \\
Location & 15 & 1 \\
Femur & 6 & 2 \\
Tibia & 3 & 1 \\
Humerus & 1 & 0 \\
Fibula & 4 & \\
Other & & 6 \\
Histology & & 3 \\
Osteoblastic & 16 & 1 \\
Chondroblastic & 4 & 1 \\
Fibroblastic & 4 & \\
Telangiectatic & 2 & \\
Metastasis & & \\
Present & & \\
Absent & & \\
\hline
\end{tabular}

${ }^{a}$ MTAP expression was detected by IHC.

Methylation-specific PCR was performed exactly as previously described (18). Aliquots of PCR products were electrophoresed in $2 \%$ agarose gels and visualized by ethidium bromide staining.

Cell proliferation assay. Osteosarcoma cell lines (SaOS-2, U2OS, HOS, KHOS, MG63 and G292) and transfectomas were incubated in wells containing $1 \times 10^{3}$ cells per $100 \mu 1$ of medium for $24 \mathrm{~h}$, using 96-well cell culture microplates (Corning Incorporated Life Sciences, Lowell, MA, USA). After 24-h incubation, the medium was changed to that containing various concentrations of the test drugs (MTX and L-alanosine), followed by incubation for $72 \mathrm{~h}$. Then, $10 \mu \mathrm{l}$ of 3-(4,5-dimethylthiazol-2-yl)-2,5-diphenyltetrazolium bromide (MTT, $5 \mathrm{mg} / \mathrm{ml}$; Sigma) was added. Following incubation for $4 \mathrm{~h}$ at $37^{\circ} \mathrm{C}$, formazan that formed was extracted with $100 \mu \mathrm{l}$ of acid-isopropanol for $24 \mathrm{~h}$. The optical density was measured at a wavelength of $595 \mathrm{~nm}$.

\section{Results}

Patient characteristics. The clinical characteristics of 40 Japanese osteosarcoma patients are recapitulated in Table I. The age of patients at diagnosis was 6-79 years (median: 17.5), with a gender ratio of 21 males to 19 females. The 


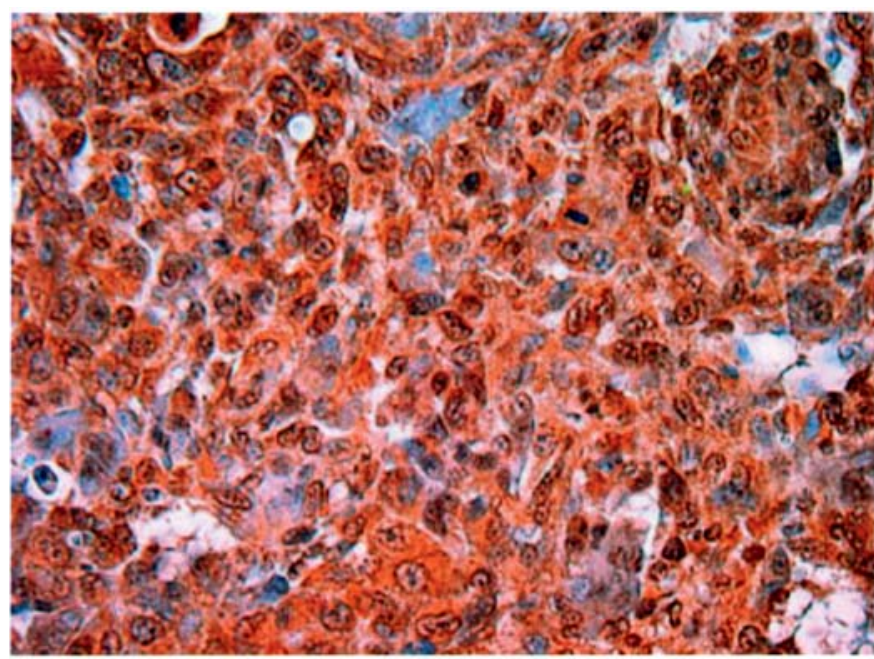

(A)

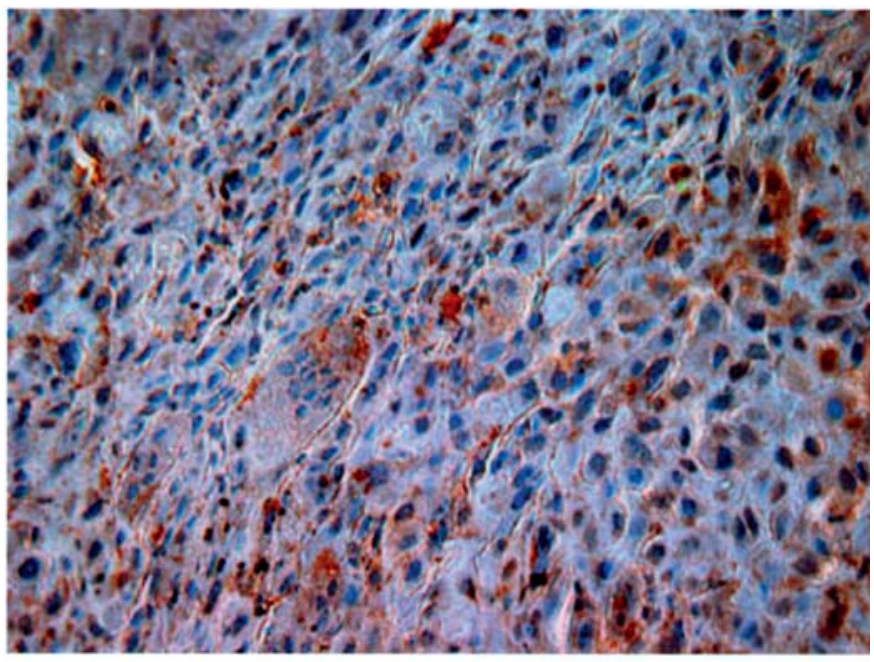

(B)

Figure 1. Detection of MTAP protein in MTAP-positive (A) and -negative (B) osteosarcomas with immunostaining. Each specimen was stained as described in the Materials and methods section. All stained slides were microscopically observed with x400 magnification and examined independently by two pathologists.

(A)
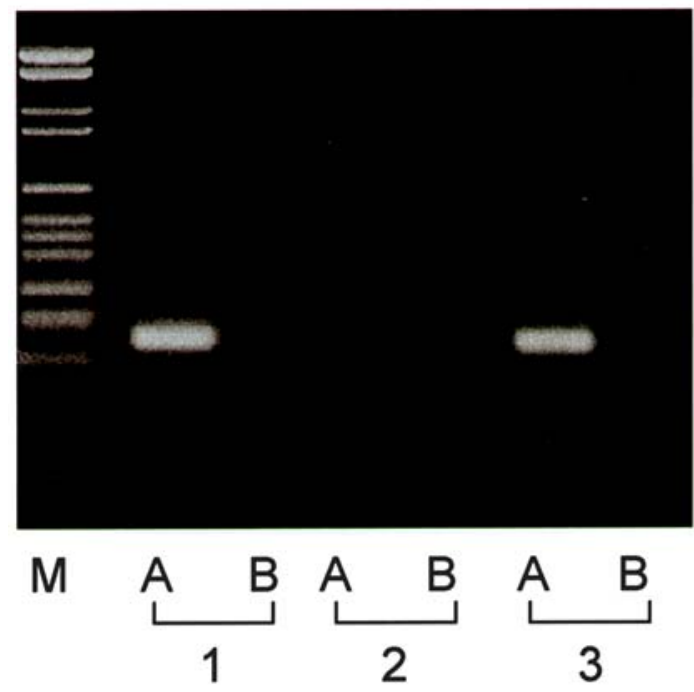

(B)

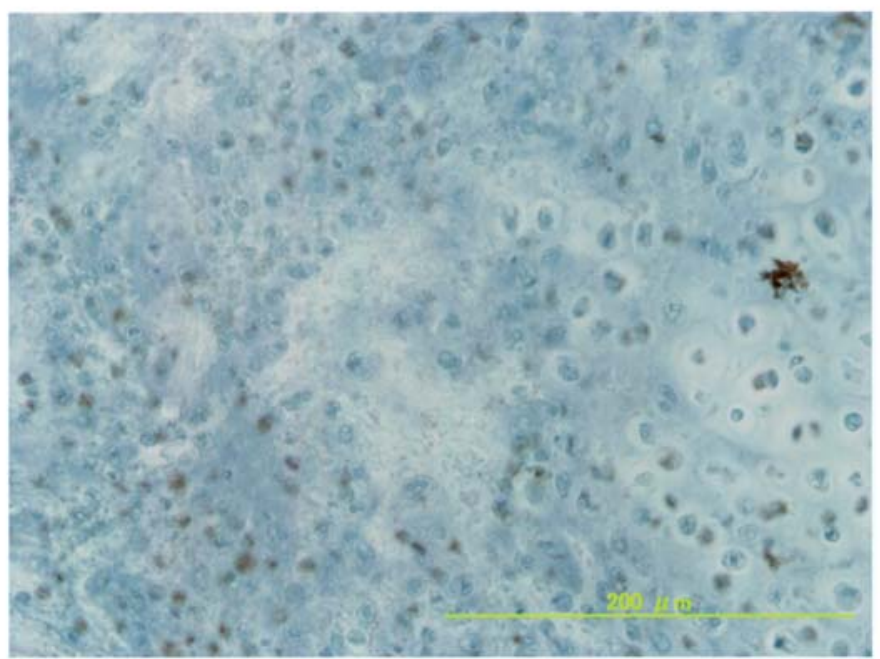

Figure 2. Analysis of samples having the intact MTAP gene but no protein by methylation-specific PCR (A) and immunohistochemistry (B). Three samples (patient no. 1, 8, and 12 in Table III) were analyzed by methylation-specific PCR. In two samples (no. 1 and 12) the promoter was methylated (A). In the sample from patient no. 8, MTAP protein was not expressed although the promoter was not methylated (B).

anatomic sites of the primary osteosarcoma included femur (22 patients), tibia (7 patients), humerus (5 patients), fibula ( 2 patients) and others (4 patients). All were histologically classified as conventional type (22 osteoblastic, 10 chondroblastic, and 5 fibroblastic cases) and telangiectatic type (3 cases). Only $6(15 \%)$ of 40 patients had remote metastasis at diagnosis.

MTAP expression in osteosarcoma patients. The MTAP expression in 40 Japanese osteosarcoma patients was analyzed immunohistochemically as described in the Materials and methods. Since the MTAP-positive osteosarcoma showed cytoplasmic staining (Fig. 1A), the MTAP expression was determined according to the criteria described in Materials and methods. As shown in Fig. 1B, the specimen having the immunostained area $<25 \%$ was regarded as negative. The MTAP expression was negative in 11 (27.5\%) of 40 osteosarcoma patients. No significant differences were found between MTAP expression and clinical data such as age, sex, histological type and metastatic status (Table II).

Analysis of the mechanism of MTAP deficiency. In order to analyze the mechanism of MTAP deficiency in osteosarcoma, genomic DNAs were extracted from the tumor samples and 
Table III. MTAP status: Comparison of IHC with the quantitative RT-PCR.

\begin{tabular}{lccc}
\hline $\begin{array}{l}\text { Patient } \\
\text { number }\end{array}$ & Immunohistochemistry & RT-PCR $^{\mathrm{a}}$ & Methylation \\
\hline 1 & & & \\
2 & - & 0.93 & + \\
3 & + & 1.00 & \\
4 & + & 0.78 & \\
5 & + & 0.72 & \\
6 & + & 0.90 & \\
7 & + & 0.72 & \\
8 & + & 0.79 & \\
9 & - & 1.00 & - \\
10 & - & 0.17 & N.D. \\
11 & + & 0.86 & \\
12 & - & 0.76 & + \\
13 & - & 0.10 & N.D. \\
14 & - & 0.47 & N.D. \\
15 & + & 1.07 & \\
\hline
\end{tabular}

${ }^{a}$ The ratio of the MTAP gene to pseudogene was calculated as described in Materials and methods. N.D., not done.

were subjected to the quantitative RT-PCR and to the methylation-specific PCR. Only fifteen of forty osteosarcoma samples were suitable for DNA extraction. DNA samples were analyzed for MTAP gene deletion. Of 15 samples, 6 tested negative with IHC (patient no. 1, 8, 9, 11, 12, and 13 in Table III). RT-PCR assay was performed independently and identified 3 samples whose MTAP gene was deleted (patient no. 9, 12, and 13 in Table III). All three samples with MTAP gene deletion were negative with IHC. The remaining 3 samples (patient no. 1, 8, and 11 in Table III) without MTAP expression by IHC had a ratio of MTAP gene to MTAP pseudogene of $0.93,1.00$, and 0.76 , respectively, indicating the presence of an intact MTAP gene based on the cut-off value of 0.6 for the gene deletion. These three were analyzed by methylation-specific PCR. As shown in Fig. 2A, two samples were found to have the methylated promoter, although one sample (patient no. 8) was not methylated. The sample from patient no. 8 was strongly negative with IHC (Fig. 2B).

We also analyzed 6 osteosarcoma cell lines by Western blot analysis. As shown in Fig. 3, two cell lines, SaOS-2 and U2OS, had MTAP protein, whereas 4 cell lines, HOS, MG63, KHOS, and G292, lacked the immunoreactive protein. The quantitative RT-PCR analysis showed that four cell lines lacking MTAP protein had deletion of the MTAP gene (data not shown).

Generation of a transfectoma expressing MTAP. The MTAP expression vector and the control vector having no insert were used to transfect four MTAP-negative osteosarcoma cell lines. After transfection with Lipofectamine 2000 and selection with Zeocin as described in Materials and methods,

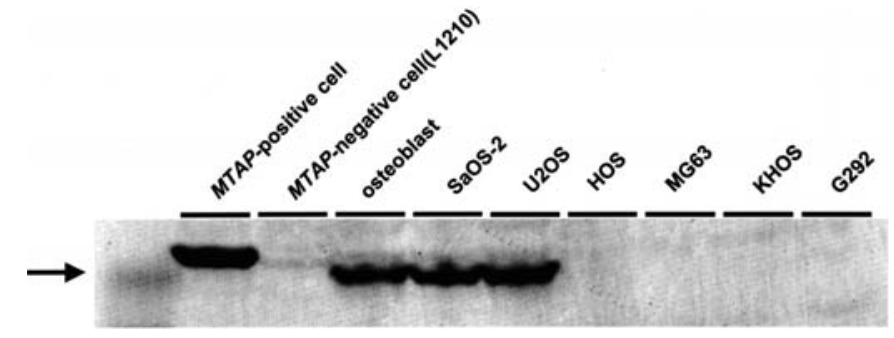

Figure 3. Western blot analysis of osteosarcoma cell lines. Western blot analysis was performed as described in the Materials and methods section. L1210 cells transfected with the MTAP cDNA expressing plasmid shown as MTAP-positive control cell and osteoblasts (hFOB) served as positive controls, wheras L1210 cells were the negative control.

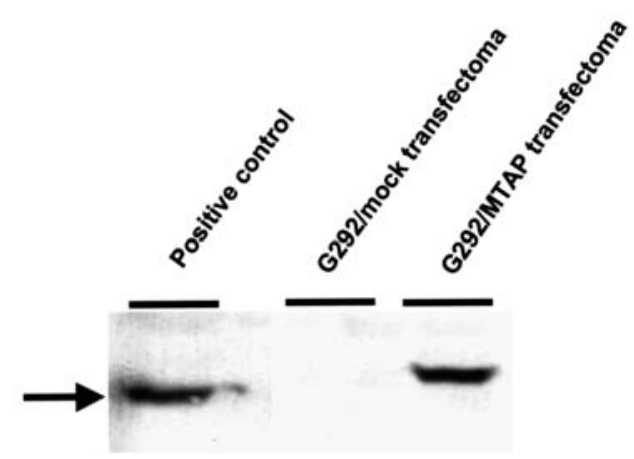

Figure 4. Western blot analysis of transfectomas. Transfectomas were generated by introducing MTAP cDNA or mock vectors into MTAP-negative osteosarcoma cell line G292. The resultant transfectomas were analyzed by Western blotting. Positive control, osteoblast (hFOB); G292/mock transfectoma, G292 transfected with the mock vector; G292/MTAP transfectoma, G292 transfected with the MTAP cDNA vector.

Zeocin-resistant clones were obtained. Individual clones were further expanded and analyzed for MTAP expression by Western blotting. A transfectoma expressing MTAP (G292/ MTAP transfectoma) was obtained from the G292 osteosarcoma cell line and used for further analysis along with the mock transfectoma (G292/mock transfectoma) (Fig. 4).

Chemotherapy targeting MTAP deficiency in osteosarcoma cell lines and transfectoma. Six osteosarcoma cell lines were cultured in the presence of the inhibitors of AMP synthesis (MTX and L-alanosine). The cell viability was estimated by MTT assay. The MTAP status influenced the chemosensitivity of the cell lines to both inhibitors of AMP synthesis (Fig. 5). The $\mathrm{IC}_{25}$ of MTX for MTAP-positive cell lines was $>200 \mu \mathrm{M}$, while that for MTAP-negative cell lines ranged from 1 to $10 \mu \mathrm{M}$ (Fig. 5A). The other inhibitor, L-alanosine, was more potent against MTAP-negative cell lines, with an $\mathrm{IC}_{25}<1 \mu \mathrm{M}$, although the $\mathrm{IC}_{25}$ for MTAP-positive cell lines was $>10 \mu \mathrm{M}$. When cell lines were treated with L-alanosine at $25 \mu \mathrm{M}$, the cell viability of MTAP-negative and MTAP-positive cell lines was $<0.2$ and $>0.5$, respectively, indicating that L-alanosine can selectively kill MTAP-negative cancers (Fig. 5B).

Since these osteosarcoma cell lines have different genetic background other than MTAP status, the difference in the chemosensitivity between MTAP-positive and MTAP-negative cell lines may not be solely due to MTAP deficiency. To clarify 
(A)

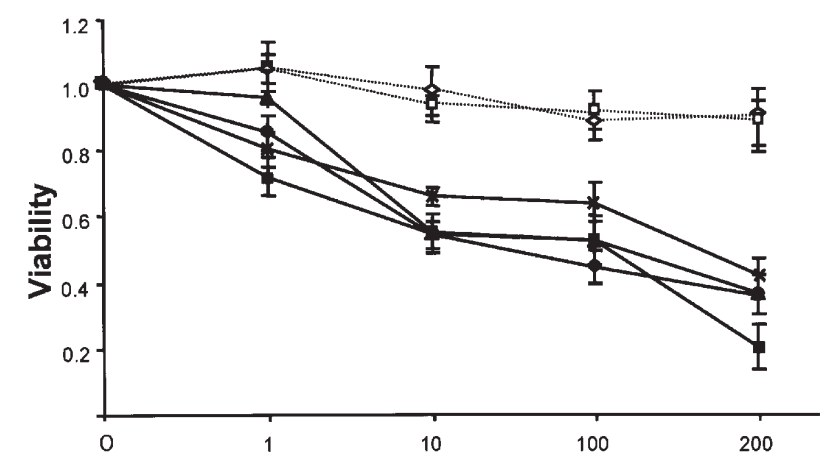

$\operatorname{MTX}(\mu \mathrm{M})$
(B)

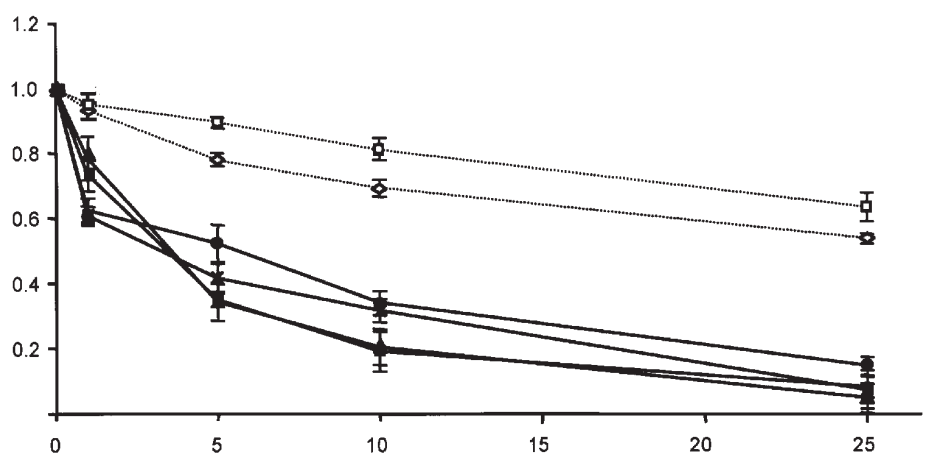

L-Alanosine ( $\mu \mathrm{M})$

Figure 5. Chemosensitivity of osteosarcoma cell lines to MTX (A) or L-alanosine (B). Osteosarcoma cell lines were cultured in the presence of MTX or L-alanosine at the indicated concentrations. The results are shown as mean \pm S.D. of three independent experiments. $\square$, MTAP-positive U2OS; $\diamond$, MTAPpositive SaOS-2; $\mathbf{\wedge}$, MTAP-negative KHOS; • MTAP-negative HOS; $\boldsymbol{\bullet}$, MTAP-negative G292; X, MTAP-negative MG63.

(A)

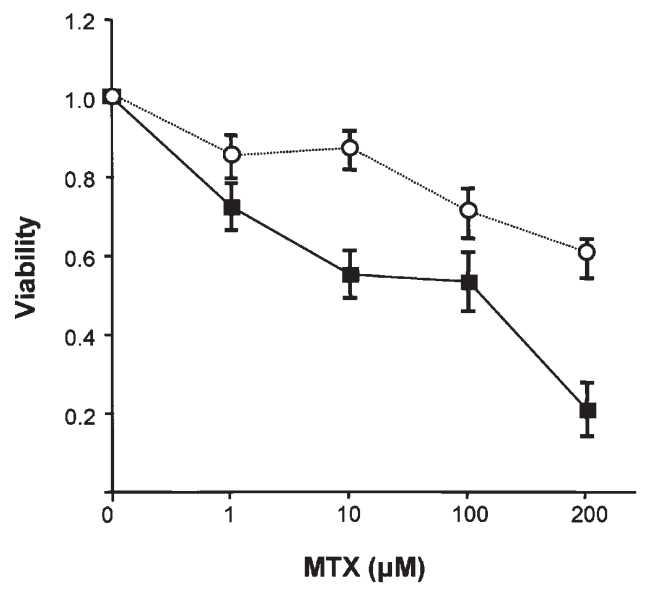

(B)

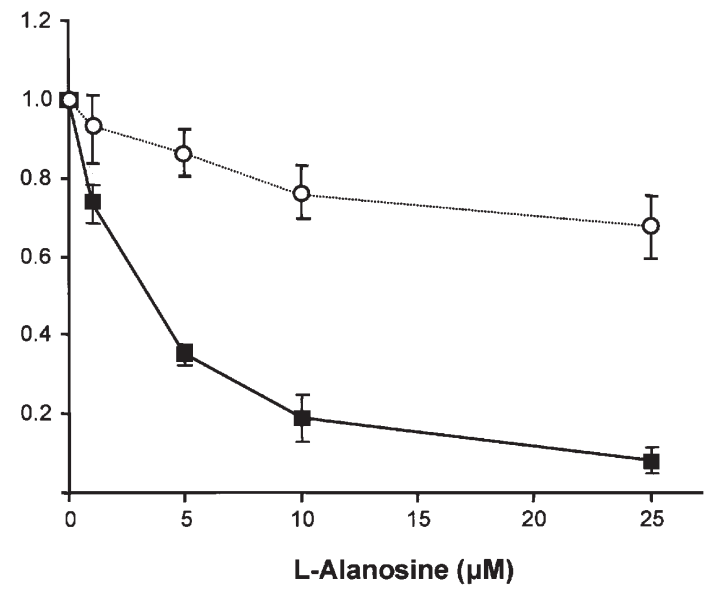

Figure 6. Chemosensitivity of transfectomas to MTX (A) or L-alanosine (B). Transfectomas generated from G292 were incubated with drugs at the indicated concentrations as described in the Materials and methods section. The results are the mean \pm S.D. of three independent experiments. $\circ$, G292/MTAP transfectoma; - G292/mock transfectoma.

this possibility, osteosarcoma cell lines were genetically engineered to express MTAP by transfection with the MTAP expression vector. Transfectomas generated from MTAPnegative cell line G292 were used for the chemosensitivity experiments (Fig. 6). G292/MTAP transfectoma expressing MTAP and G292/mock transfectoma not expressing MTAP were treated with either MTX or L-alanosine. The difference in chemosensitivity based on the enzyme deficiency was more evident in the transfectoma system than in the cell lines. It is of note that L-alanosine was able to selectively kill G292/mock transfectoma compared to G292/MTAP transfectoma. With MTX, the selectivity of the chemotherapy was not remarkable, partly because MTX inhibits both purine and pyrimidine biosynthesis. Although G292/MTAP transfectoma is not an exact replica of normal cells, it is very similar to normal cells in terms of MTAP status. Accordingly, the results with transfectomas suggest that MTAP deficiency can be utilized for selective chemotherapy.

\section{Discussion}

MTAP cleaves MTA into adenine and MTR-1-P, which are converted to AMP and methionine, respectively $(1,2)$. In the previous studies, MTAP was found to be an enzyme ubiquitously expressed in all normal tissues, but absent in a variety of malignant tumors such as glioma, non-small cell lung cancer, bladder cancer, malignant melanoma, pancreatic cancer, adult T-cell leukemia (ATL) and osteosarcoma (5-15).

The human MTAP gene is mapped on chromosome 9p21 and is in close proximity to the tumor suppressor genes p16 $16^{\mathrm{INK} 4 \mathrm{a}}$ and $\mathrm{p} 15^{\mathrm{INK} 4 \mathrm{~b}}$. Frequent genetic or epigenetic alternations are observed in the region harboring these genes, resulting in the loss of and expression of protein. MTAP deficiency is mainly caused by deletion of the MTAP gene (23), which is often co-deleted with these tumor suppressor genes. The MTAP gene contains eight exons and seven introns. The previous reports showed that the last 8th exon of the MTAP gene was 
always deleted even when the gene deletion was partial. It has been observed that promoter hypermethylation and histone acetylation/deacetylation down-regulated the gene expression via the epigenetic gene silencing mechanisms in cancer $(29,30)$. Recently we and another group reported that MTAP deficiency was caused by the promoter hypermethylation in a cultured cell line (18) and in hepatocellular carcinoma (19).

For osteosarcoma, the effective drugs, such as doxorubicin, cisplatin, methotrexate, and ifosfamide have remained the same for over 20 years. The combination of surgery and adjuvant or neoadjuvant chemotherapy improved the 5-year survival rate from $10 \%$ to $70 \%$, although the 5-year survival rate is $20-30 \%$ in patients with metastasis at diagnosis (1). Drugs targeting the molecular characteristics have been developed and used for chronic myelogenous leukemia and non-small cell lung cancer $(31,32)$. MTAP deficiency is a known molecular target for chemotherapy in a variety of cancers. Garcia-Castellano et al suggested that the high frequency of MTAP deficiency in primary osteosarcoma might be a target for treatment (17). They analyzed 96 samples of high-grade osteosarcoma and found MTAP gene deletions in $36(37.5 \%)$. Our study revealed that MTAP protein was negative with IHC in $11(27.5 \%)$ of 40 samples. As reported here, the frequency of MTAP deficiency in Japanese osteosarcoma patients was lower than that in Caucasian patients. This might be due to the fact that metastasis at diagnosis was present in $25 \%$ of patients in the previous report (17) compared to $15 \%$ of patients in our study. This speculation is also supported by the finding that the MTA gene was lost during the progression of disease in four cases. Garcia-Castellano et al did not mention mechanisms other than gene deletions, although an epigenetic mechanism might have caused MTAP deficiency in one case which showed no MTAP protein or mRNA despite the intact MTAP gene in their study (17). We analyzed a subset of 15 samples from which genomic DNAs were available for MTAP gene deletions and promoter hypermethylation. Out of 15, 6 samples were negative with IHC. MTAP gene deletion was found in 3 samples, while the MTAP promoter was methylated in 2 samples. These five samples tested negative with IHC. One sample (patient no. 8 in Table III) was negative with IHC, although neither gene deletion nor promoter hypermethylation was detected (Table III, Fig. II). These data indicated that MTAP deficiency was caused not only by gene deletions but also by epigenetic mechanisms such as promoter hypermethylation and histone modifications $(29,30,33)$. Accordingly, MTAP deficiency should be diagnosed based on the absence of MTAP protein expression, although there are several diagnostic methods for MTAP deficiency such as MTAP enzyme assay, Western blot analysis, fluorescence in situ hybridization (FISH), quantitative PCR, and Southern blot analysis $(2,8,16,24,25)$.

In tumor cells lacking MTAP enzyme, purine nucleotides completely depend on de novo synthesis, since adenine is not produced endogenously from MTA in these cells (2). Therefore, the MTAP-negative cells are not rescued from the toxicity of the inhibitors of de novo AMP synthesis such as methotrexate (MTX), dideazatetrahydrofolate (DDATHF) and L-alanosine, while the MTAP-positive normal cells utilize adenine derived from MTA to overcome the purine-deficient state induced by these inhibitors $(9,15)$. Based on the biochemical difference between MTAP-positive normal cells and MTAP-negative tumor cells, selective chemotherapy targeting MTAP deficiency has been tested in cell culture systems using T-cell acute lymphoblastic leukemia (T-ALL) cell lines $(20,23)$ and adult T-cell leukemia (ATL) cell lines (22) and in primary T-ALL cells (34). Hori et al determined the role of MTAP in the chemosensitivity to inhibitors of purine de novo synthesis using the MTAP-negative lung cancer cell line A549 either transfected with MTAP sense or antisense expression vectors (26).

In the previous report of MTAP deficiency in osteosarcoma, the chemosensitivity of MTAP-negative osteosarcoma cell lines to inhibitors of purine de novo synthesis was not determined (17). In this study, therefore, six osteosarcoma cell lines were tested for sensitivity to MTX or L-alanosine and for MTAP protein expression by Western blotting. As shown in Figs. 3 and 4, chemosensitivity to MTX and L-alanosine was attributable to MTAP deficiency. One could argue that the difference in chemosensitivity might be due to factors other than MTAP deficiency. We generated MTAP expressing transfectoma from MTAP-negative G292 cells along with mock transfectoma and tested the sensitivity to MTX or Lalanosine (Figs. 5 and 6). MTAP-negative mock transfectoma was significantly sensitive to both inhibitors compared with MTAP expressing transfectoma and this tendency was more evident with L-alanosine. As reported in other tumor types, the MTAP deficiency found in osteosarcoma can be exploited for chemotherapy with inhibitors of purine de novo synthesis.

In summary, we analyzed the MTAP deficiency in Japanese osteosarcoma patients and found that this enzyme deficiency was caused by genetic and epigenetic mechanisms. We also found that IHC was the best and reliable diagnostic method for MTAP deficiency irrespective of mechanisms involved in the enzyme deficiency. Moreover, we demonstrated that MTAP deficiency could be exploited for selective chemotherapy for MTAP-negative osteosarcoma with inhibitors of purine de novo synthesis.

\section{Acknowledgements}

The authors would like to thank Ms. C. Usui for her excellent secretarial assistance. This study was supported in part by a Grant-in-Aid from the Ministry of Education, Culture, Sports, Science and Technology of Japan.

\section{References}

1. Longhi A, Errani C, De Paolis M, Mercuri M and Bacci G: Primary bone osteosarcoma in the pediatric age: state of the art. Cancer Treat Rev 32: 423-436, 2006.

2. Kamatani N, Nelson-Rees WA and Carson DA: Selective killing of human malignant cell lines deficient in methylthioadenosine phosphorylase, a purine metabolic enzyme. Proc Natl Acad Sci USA 78: 1219-1223, 1981.

3. Toohey JI: Methylthioadenosine nucleoside phosphorylase deficiency in methylthio-dependent cancer cells. Biochem Biophys Res Commun 83: 27-35, 1978.

4. Nobori T, Takabayashi K, Wu DJ, Tran P, Orvis L, Batova A, Yu AL and Carson DA: Genomic cloning of methylthioadenosine phosphorylase deficiency: a purine metabolic enzyme deficient in multiple different cancers. Proc Natl Acad Sci USA 93: 6203-6208, 1996.

5. Nobori T, Miura K, Wu DJ, Lois A, Takabayashi K and Carson DA: Deletion of the cyclin-dependent kinase-4 inhibitor gene in multiple human cancers. Nature 368: 753-756, 1994. 
6. Olopade OI, Pomykala HM, Hagos F, Sveen LW, Espinosa R III, Dreyling MH, Gursky S, Stadler WM, LeBeau MW and Bohlander SK: Construction of a 2.8 megabase yeast artificial chromosome contig and cloning of the human methylthioadenosine phosphorylase gene from the tumor suppressor region on 9p21. Proc Natl Acad Sci USA 92: 6489-6493, 1995.

7. Kamatani N, Yu AL and Carson DA: Deficiency of methylthioadenosine phosphorylase in human leukemic cells in vivo. Blood 60: 1387-1391, 1982 .

8. Nobori T, Karras JG, Fulvio DR, Waltz TA, Pojen PC and Carson DA: Absence of methylthioadenosine phosphorylase in human gliomas. Cancer Res 51: 3193-3197, 1991.

9. Nobori T, Szinai I, Amox D, Parker B, Olopade OI, Buchhagen DL and Carson DA: Methylthioadenosine phosphorylase deficiency in human non-small cell lung cancers. Cancer Res 53: 1098-1101, 1993.

10. Fitchen JH, Riscoe MK, Dana BW, Lawrence HJ and Ferro AJ: Methylthioadenosine phosphorylase deficiency in human leukemias and solid tumors. Cancer Res 46: 5409-5412, 1986.

11. Traweek ST, Riscoe MK, Ferro AJ, Braziel RM, Magenis RE and Fitchen JH: Methylthioadenosine phosphorylase deficiency in acute leukemia: pathologic, cytogenetic, and clinical features. Blood 71: 1568-1573, 1988 .

12. Stadler WM, Sherman J, Bohlander SK, Roulston D, Dreyling M, Rukstalis D and Olopade OI: Homozygous deletions within chromosomal bands 9p21-22 in bladder cancer. Cancer Res 54: 2060-2063, 1994.

13. Smaaland R, Schanche JS, Kvinnsland S, Hostmark J and Ueland PM: Methylthioadenosine phosphorylase in human breast cancer. Breast Cancer Res Treat 9: 53-59, 1987.

14. Cowan JM, Halaban R and Francke U: Cytogenetic analysis of melanocytes from premalignant nevi and melanomas. J Natl Cancer Inst 80: 1159-1164, 1988.

15. Chen ZH, Zhang H and Savarese TM: Gene deletion chemoselectivity codeletion of the genes for $\mathrm{p} 16^{\mathrm{INK} 4}$, methylthioadenosine phosphorylase, and the $\alpha$ - and $\beta$-interferons in human pancreatic cell carcinoma lines and its implications for chemotherapy. Cancer Res 56: 1083-1090, 1996.

16. Hori Y, Hori H, Yamada Y, Carrera CJ, Tomonaga M, Kamihira S, Carson DA and Nobori T: The methylthioadenosine phosphorylase gene is frequently co-deleted with p16INK4a gene in acute type adult T-cell leukemia. Int J Cancer 75: 51-56, 1998.

17. Garcia-Castellano JM, Villanueva A, Healey JH, Sowers R, Cordon-Cardo C, Huvos A, Bertino JR, Meyers P and Gorlick R: Methylthioadenosine phosphorylase gene deletions are common in osteosarcoma. Clin Cancer Res 8: 782-787, 2002.

18. Ishii M, Nakazawa K, Wada H, Nshioka J, Nakatani K, Yamada Y, Kamihira S, Kusunoki $M$ and Nobori T: Methylthioadenosine phosphorylase gene is silenced by promoter hypermethylation I human lymphoma cell line DHL-9: Another mechanism of enzyme deficiency. Int J Oncol 26: 985-991, 2005.

19. Hellerbrand C, Mühlbauer M, Wallner S, Schuierer M, Behrmann I, Bataille F, Weiss T, Schölmerich J and Bosserhoff AK: Promoter-hypermethylation is causing functional relevant downregulation of methylthioadenosine phosphorylase (MTAP) expression in hepatocellular carcinoma. Carcinogenesis 27: 64-72, 2006.
20. Batova A, Dicciannni MB, Nobori T, Vu T, Yu J, Bridgeman L and $\mathrm{Yu}$ A: Frequent deletion in the methylthioadenosine phosphorylase gene in T-cell acute lymphoblastic leukemia: strategies for enzyme-targeted therapy. Blood 88: 3083-3090, 1996.

21. Yu J, Batova A, Shao L, Carrera CJ and Yu AL: Presence of methylthioadenosine phosphorylase (MTAP) in hematopoietic stem/progenitor cells: its therapeutic implication for MTAP (-) malignancies. Clin Cancer Res 3: 433-438, 1997.

22. Harasawa H, Yamada Y, Kudoh M, Sugahara K, Soda H, Hirakata Y, Sasaki H, Ikeda S, Matsuo T, Tomonaga M, Nobori T and Kamihira S: Chemotherapy targeting methylthioadenosine phosphorylase (MTAP) deficiency in adult T cell leukemia (ATL). Leukemia 16: 1799-1807, 2002.

23. Efferth T, Miyachi H, Drexler HG and Gebhart E: Methylthioadenosine phosphorylase as target for chemoselective treatment of T-cell acute lymphoblastic leukemic cells. Blood Cells Mol Dis 28: 47-56, 2002.

24. Perry A, Nobori T, Ru N, Anderl K, Borell TJ, Mohapatra G, Feuerstein BG, Jenkins RB and Carson DA: Detection of p16 gene deletions in gliomas: a comparison of fluorescence in situ hybridization (FISH) versus quantitative PCR. J Neuropath Exp Neurol 56: 999-1008, 1997.

25. M'soka TJ, Nishioka J, Taga A, Kato K, Kawasaki H, Yamada Y, Yu A, Komada Y and Nobori T: Detection of methylthioadenosine phosphorylase (MTAP) and p16 gene deletion in T cell acute lymphoblastic leukemia by real-time quantitative PCR assay. Leukemia 14: 935-940, 2000.

26. Hori H, Tran P, Carrera CJ, Hori Y, Rosenbach MD, Carson DA and Nobori T: Methylthioadenosine phosphorylase cDNA transfection alters sensitivity to depletion of purine and methionine in A549 lung cancer cells. Cancer Res 56: 5653-5658, 1996.

27. Galfre G and Milstein C: Preparation of monoclonal antibodies: strategies and procedures. Methods Enzymol 73: 3-46, 1981

28. Harlow E and Lane D (eds): Antibodies: a laboratory manual. Cold Spring Harbor Laboratory. Cold Spring Harbor, NY, 1988.

29. Herman JG, Graff JR, Myohanen S, Nelkin BD and Baylin SB Methylation-specific PCR: a novel PCR assay for methylation status of CpG islands. Proc Natl Acad Sci USA 93: 9821-9826, 1996.

30. Herranz M and Esteller M: DNA methylation and histone modifications in patients with cancer: potential prognostic and therapeutic targets. Methods Mol Biol 361: 25-62, 2007.

31. Cohen MH, Williams GA, Sridhara R, Chen G and Pazdur R: FDA drug approval summary: gefitinib (ZD1839) (Iressa) tablets. Oncologist 8: 303-306, 2003

32. Druker BJ, Tamura S, Buchdunger E, Ohno S, Segal GM, Fanning S, Zimmermann J and Lydon NB: Effects of a selective inhibitor of the Abl tyrosine kinase on the growth of Bcr-Abl positive cells. Nat Med 2: 561-566, 1996.

33. Verdone I, Caserta M and Di Mauro F: Role of histone acetylation in the control of gene expression. Biochem Cell Biol 83: 344-353, 2005.

34. Batova A, Diccianni MB, Omura-Minamisawa M, Yu J, Carrera CJ, Bridgeman LJ, Kung FH, Pullen J, Amylon MD and Yu AL: Use of alanosine as methylthioadenosine phosphorylaseselective therapy for T-cell acute lymphoblastic leukemia in vitro. Cancer Res 59: 1492-1497, 1999. 